\title{
Systematic review and meta-analysis of post-transplant hepatic artery and biliary complications in patients treated with trans- arterial chemoembolization before liver transplantation
}

\section{$\underline{\text { Authors }}$}

Dimitri Sneiders ${ }^{1, *}$, Thymen Houwen ${ }^{1, *}$, Liset H.M. Pengel ${ }^{2}$, Wojciech G. Polak ${ }^{1}$, Frank J.M.F. Dor ${ }^{1,3}$, Hermien Hartog ${ }^{1}$

\section{$\underline{\text { Affiliations }}$}

* Both authors contributed equally

${ }^{1}$ Department of Surgery, Division of Hepato-pancreato-biliary and Transplant Surgery, Erasmus MC University Medical Center, Rotterdam, the Netherlands

${ }^{2}$ Peter Morris Centre for Evidence in Transplantation, Nuffield Department of Surgical Sciences, University of Oxford, Oxford, UK

${ }^{3}$ West London Renal and Transplant Centre, Hammersmith Hospital, Imperial College Healthcare NHS Trust, London, UK

\section{Corresponding author}

Hermien Hartog, HPB/Transplant surgeon

Dept of Surgery, Erasmus MC, suite H-0818k

P.O. Box 2040,

3000 CA Rotterdam, The Netherlands

h.hartog@erasmusmc.nl 


\section{Author Contributions:}

TH, DS and HH designed the study, collected data, and wrote the manuscript, LHMP and FJFMD contributed to conception and design of the review, all authors contributed by intellectual content and critical review of the manuscript.

The authors declare no conflicts of interest

Prospero registration: CRD42016025734.

\section{Keywords:}

Transarterial Chemoembolization

Hepatocellular carcinoma

Liver transplantation

Hepatic artery thrombosis

Biliary tract

Postoperative complications 


\section{Abbreviations:}

AS, anastomotic strictures

CI, confidence interval

ESOT, European Society for Organ Transplantation

HCC, hepatocellular carcinoma

NAS, non-anastomotic strictures

NR, not reported

NS, not specified

OR, odds ratio

PMCET, Peter Morris Centre for Evidence in Transplantation

PRISMA, Preferred Reporting Items for Systematic Reviews and Meta-Analyses

TACE, trans-arterial chemoembolization 


\section{$\underline{\text { Abstract }}$}

BACKGROUND: Hepatic artery complications are feared complications after liver transplantation and may compromise the biliary tract, graft and patient survival. The objective of this systematic review and meta-analysis was to compare risk of hepatic artery and biliary complications after liver transplantation in patients who underwent neo-adjuvant trans-arterial chemoembolization (TACE) vs. no TACE.

METHODS: Comprehensive searches were performed in Embase, MEDLINE OvidSP, Web of Science, Google Scholar and Cochrane databases to identify studies concerning hepatocellular cancer patients undergoing pre-liver transplantation TACE. Quality assessment of studies was done by the validated checklist of Downs and Black. Meta-analyses were performed to evaluate the incidence of all hepatic artery complications, hepatic artery thrombosis and biliary tract complications, using binary random-effect models. Prospero registration: CRD42016025734.

RESULTS: Fourteen retrospective studies, representing 1122 TACE patients, met the inclusion criteria. Postoperative hepatic artery complications consisted of hepatic artery thrombosis, stenosis and (pseudo)-aneurysms. Pre-liver transplantation TACE was significantly associated with occurrence of post- transplant hepatic artery complications (OR: 1.57, 95\%CI 1.09-2.26, p 0.02). No significant association between neo-adjuvant TACE and hepatic artery thrombosis alone or biliary tract complications was found.

DISCUSSION: Patients treated with transarterial chemoembolization before liver transplantation may be at increased risk for development of hepatic artery complications after liver transplantation. 


\section{Introduction}

Timely arterial reperfusion is a critical step in liver transplantation. Technical difficulties and delays to re-vascularise the hepatic artery during the procedure poses a risk of critical ischemic damage to the biliary tree of the liver graft. Post-transplant compromised hepatic artery flow is a potentially devastating event, and hepatic artery thrombosis is a feared complication leading to loss of the liver graft or death in respectively $53 \%$ and $33 \%$ of hepatic artery thrombosis cases..$^{1,2}$

Trans-arterial chemoembolization (TACE) is one of the loco-regional therapies used in patients with hepatocellular carcinoma (HCC) listed for liver transplantation to bridge the gap between enlistment and transplantation, to prevent progression while waiting for a liver graft, or to downstage patients that progressed outside Milan criteria. ${ }^{2}$

No universally accepted protocol for the application of TACE exists. The treatment concept is to administer a high dose of chemotherapeutic drugs in the hepatic arteries supplying the tumour, followed by embolization of these vessels. ${ }^{3}$ TACE can be administered into the tumour, liver segment or liver lobe, nowadays selective administration of TACE is preferred. ${ }^{4}$ The chemotherapeutic drugs and combination of drugs used for TACE vary and include mono, double and triple therapies. ${ }^{3}$ For embolization both permanent and temporary embolization devices are used. ${ }^{5}$ Drug-eluting beads used in TACE combine the two functions of a drug delivery system and embolic agent.$^{6}$

TACE may cause a number of direct complications such as hepatic failure, post embolization syndrome, abscess formation, access site injury, and pulmonary embolism. ${ }^{7-9}$ Intra-arterial manipulation of the hepatic artery prior to liver transplantation as part of neo-adjuvant TACE for HCC has raised concerns of increasing risk of arterial and biliary complications posttransplantation. The incidence of arterial and/or biliary complications after liver transplantation in patients treated with TACE has been explored in observational studies, 
however the majority of these studies lack power to detect a clinically significant difference. ${ }^{10-23}$ We aimed to investigate the impact of TACE prior to liver transplantation on occurrence of hepatic artery and biliary tract complications after liver transplantation by pooled analyses of published cohorts. 


\section{Methods}

The Cochrane Handbook for Interventional Systematic Reviews was followed to conduct the systematic review and meta-analysis. ${ }^{24}$ The manuscript was written according to the PRISMA (Preferred Reporting Items for Systematic Reviews and Meta-Analyses) statement. ${ }^{25}$ The systematic review protocol was registered prospectively in PROSPERO (https://www.crd.york.ac.uk/PROSPERO, protocol number: CRD42016025734).

Comprehensive searches were performed in Embase, MEDLINE OvidSP, Web of Science, Google Scholar and the Cochrane databases. The search was performed for articles written in English and published until March 2016, concerning patients with HCC waitlisted for liver transplantation. Included papers studied post-transplant complications of the hepatic artery or biliary tract, in patients treated with TACE prior to deceased or living donor liver transplantation, compared with liver transplantation recipients who had not undergone TACE. We included papers describing trans-arterial embolization or chemo-infusion alone, embolization with drug eluting beads or chemo-embolization, and excluded papers describing radio-embolization. Case reports, letters and editorials were excluded, as well as paediatric and non-human studies. We expected most studies to be of relatively small sample size, and to achieve adequate power potentially relevant abstracts in conference proceedings were included. Search terms for each database are provided in appendix I.

Studies were independently evaluated for inclusion by two observers (TH, DS) and disagreement was resolved by consensus with a third reviewer $(\mathrm{HH})$. Studies were screened based on title and abstract first. Subsequently, full-texts were acquired if available. Finally, manual cross-referencing was performed to identify other potentially relevant studies.

Quality of selected papers was assessed using the validated checklist of Downs and Black, consisting of 27 items grouped in five sub-scales (quality of reporting, external validity, 
potential of bias, confounding, and power analysis). ${ }^{26}$ The maximum score is 32 points. Item 27 (power analysis) was modified as we anticipated a majority of observational studies in which it would be rarely feasible to reach a power of $>80 \%$. Instead of the original 5-point scale, we assigned a score of " 0 " if no power calculation was provided, " 3 " if a power calculation was provided but the impact of the difference between groups used in the calculation was unclear, and " 5 " if the difference between groups was clearly defined as a clinically important difference. Additionally the risk of bias and quality of evidence categorization according to the GRADE score was applied (http://www.gradeworkinggroup.org).

Data were extracted from the full-text articles independently by two observers (TH, DS) with use of a standard extraction table, disagreement was resolved by consensus with a third reviewer $(\mathrm{HH})$. The standard extraction table included the incidence of postoperative hepatic artery (primary outcome) and biliary complications (secondary outcome), anastomotic technique, re-interventions following liver transplantation and death from hepatic artery complications, follow-up schedule, diagnostic methods, type of TACE, number of TACE procedures, type of donor, etiology of liver disease, MELD scores and tumor characteristics. If for a patient the same complication was reported multiple times, this was considered a single complication in the pooled analysis.

Meta-analyses were performed with OpenMetaAnalyst, using a binary random-effects model (DerSimonian Laird method) because we anticipated heterogeneity between studies. A 0.5 correction factor was used, if the number of events was $0 .{ }^{27}$ For each outcome, the results were presented in forest plots with an odds ratio and 95\% confidence interval (CI). A P-value $<0.05$ was considered statistically significant. Potential heterogeneity between studies was assessed with $\mathrm{I}^{2}$ tests. A sensitivity analysis was performed for the primary and secondary 
outcomes, testing for the effect of three factors: overall quality of the studies $(\geq 50 \%$ of available points), full text studies compared to abstracts and whether or not a clear definition of hepatic artery or biliary tract complications was reported in the methods section. The estimates of the sensitivity analyses were compared to the analyses including all studies with a Z-test to check for significant differences. 


\section{$\underline{\text { Results }}$}

\section{Literature search results}

Out of 4,002 records in the initial search, 14 records ( 8 full articles, 6 conference proceedings) met our inclusion criteria (Fig. 1). ${ }^{10-23}$ Combined, these retrospective studies represent a total of 1,122 patients who underwent neo-adjuvant TACE or trans arterial embolization for HCC prior to liver transplantation, and 2,703 patients not bridged with TACE. Seven studies described solely hepatic artery complications, three studies described both hepatic artery and biliary tract complications, and four studies described biliary tract complications only. A meta-analysis of hepatic artery complications comprised data of 10 studies. ${ }^{11,13-18,20,21,23}$ Meta-analysis of biliary tract complications comprised data of seven studies..$^{10-12,15,17,19,22}$ One study with a limited follow up time of 7 days was excluded from the analysis. ${ }^{28}$

\section{Quality assessment}

The quality assessment is presented in Table 1. All included studies are of observational and retrospective design. Goel et al. represented the largest cohort to date, and was the only study performing a power analysis. ${ }^{11}$ The sample size in most studies is limited ranging from 19 to 328 TACE cases per study and no study shows a large effect size. According to our quality assessment tool, all included studies are prone to confounding. Five out of eight of the full text studies obtained more than $50 \%$ of the available points (median: $17 / 32$ points, $\mathrm{N}=8$ ), compared to none of the congress abstracts (median: 12/32 points, $\mathrm{N}=6$ ).

Five studies provided a clear definition of hepatic artery complications, including thrombosis, stenosis, (pseudo)-aneurysms and anastomotic disruption established by radiological evaluation of the artery. ${ }^{11,13,16,20,23}$ Detection of arterial complications by a standardized protocol during long-term clinical follow-up by Doppler ultrasound or CT-angiography was 
reported in four studies. ${ }^{13,15,17,20}$ Four studies defined biliary tract complications clearly, which included anastomotic strictures, non-anastomotic strictures, and/or biliary leaks. ${ }^{10-12,19}$ Six studies are at risk for selection bias: In four of these studies the control group was flawed by inclusion of non-HCC cases $^{10,12,16,20}$, two studies excluded patients that died within 30 days post-operatively ${ }^{10,13}$ and one study did not clearly report their source population. ${ }^{18}$

Risk of bias from selective reporting of outcomes was identified in six studies, which did not report a definition of hepatic artery or biliary tract complications in the methods section. ${ }^{14,15,17,18,22} 21$ Therefore, the quality of all included studies according to GRADE is low to very low.

\section{Baseline Characteristics}

Table 2 shows the baseline characteristics of the individual studies. Follow-up was longer than six months in ten studies. Four studies did not report the exact follow up time. ${ }^{16,20,22,23}$

\section{Hepatic artery complications}

Data on postoperative hepatic artery complications are summarized in Table 3. Post-transplant hepatic artery complications occurred more frequently in TACE recipients (76/837) compared to non-TACE recipients (145/2294). We observed a significant association between pre-liver transplantation TACE and post transplantation occurrence of hepatic artery complications, including thrombosis, stenosis and (pseudo)-aneurysms (OR: 1.57, 95\%CI 1.09-2.26, p 0.016; $\mathrm{I}^{2}=0 \%$ ) (Fig. 2). ${ }^{11,13-18,20,21,23}$ No evidence of an association between pre-transplant TACE and post-transplant occurrence of hepatic artery thrombosis, alone, was found (OR 1.31, 95\% CI: 0.74-2.35; $\mathrm{I}^{2}=0 \%$ ) (Fig.3). ${ }^{11,14,16-18,20,21}$ There was no significant heterogeneity between studies in both analyses (Fig. 2 and Fig. 3). Series of Lin et al. was the only study reporting exclusively results of living donor liver transplantation in contrast to deceased donor liver 
transplantation, but exclusion of this study from pooled analyses, did not change the magnitude or direction of the association. ${ }^{14}$ Two other studies reported inclusion of a small proportion living donor living transplantation cases respectively, in $3 \%$ and $1.7 \%$ of the study population. ${ }^{10,11}$ Sensitivity analysis revealed that the overall estimate was not influenced by the quality of the studies, publication type or the use of a restricted definition (appendix II). Nevertheless, when restricting the analysis to full text papers, the association between preliver transplantation TACE and post-transplantation occurrence of hepatic artery complications was stronger (OR: 2.2, 95\% CI 1.3-3.6).

Time from liver transplantation to diagnosis of hepatic artery complications was reported in three studies. The median time to diagnosis was 12.5 days (range $1-120, n=14$ ) in patients treated with TACE compared to 57.5 days (range 2-91, $n=8$ ) in patients not treated with TACE. ${ }^{13,14,17}$

\section{Mechanical damage to hepatic artery after TACE}

Microscopic changes of the hepatic artery were systematically studied in three studies, and mechanical injuries to the hilar hepatic artery or anastomotic site were described in a majority of patients after TACE ${ }^{13,14,17}$. Lin et al. observed hepatic artery intima dissection in 23/40 patients $(58 \%)$ that underwent pre-liver transplantation after TAE vs. $4 / 14$ patients $(28 \%)$ in the no-TACE group. ${ }^{14}$ Two other studies reported histological injuries, such as wall edema, fibrosis, or thrombosis, at the hilar hepatic artery of explants in 12/32 (38\%) and 98/111 $(88 \%)$ of TACE pre-treated patients compared to $3 / 35(9 \%)$ and $9 / 339(3 \%)$ respectively of no-TACE patients. ${ }^{13,17}$ 
Intra-operative salvage and post-operative outcomes for hepatic artery complications

Technical data on the arterial anastomosis was reported in three studies. ${ }^{14-16}$ Intra-operative salvage by alternative arterial reconstructions or immediate revision of the arterial anastomosis was reported in 23/170 (14\%, 95\% CI: 9 - 20\%) TACE patients vs. 20/381 (5\%, 95\% CI: $3-8 \%$ ) in no-TACE patients.

The incidence of endovascular or surgical re-interventions as a result of hepatic artery complications was not consistently reported. In five studies comprising 584 TACE and 714 non-TACE patients, cumulative numbers of re-interventions related to hepatic artery complications was $42(7.2 \%, 95 \%$ CI: $5.4-9.6 \%)$ in TACE patients vs. 29 re-interventions $(4.1 \%, 95 \%$ CI: $2.8-5.8 \%)$ in non-TACE patients. ${ }^{11,13,14,17,18}$

\section{Biliary tract complications}

Data on biliary tract complications are summarized in Table 4. No strong evidence of a significant association was observed between pre-liver transplantation TACE and occurrence of biliary tract complications post-transplantation (OR 1.30 $95 \%$ CI: 0.96-1.76, p 0.087, $\mathrm{I}^{2}=0 \%$ ) (Fig. 4). ${ }^{10-13,15,17,19,22}$. There was no significant between study heterogeneity in the analyses. Sensitivity analysis revealed that the overall estimate was not influenced by the quality of the studies, publication type or the use of a restricted definition (appendix II). 


\section{$\underline{\text { Discussion }}$}

With this meta-analysis we aimed to determine whether TACE is a safe technique securing hepatic artery quality for subsequent liver transplantation in HCC patients. The relation between pre-transplantation TACE and post-transplantation arterial and biliary complications has only been explored by observational approach, limiting the evaluation of a causal relation. Majority of studies exhibited flaws in study design and reporting of data. The strength of this meta-analysis is the pooled sample size resulting in additional power to explore the evidence for an association between TACE and post-transplant arterial and biliary complications.

Although no prior study showed strong evidence of an association, our pooled meta-analysis demonstrates a significant association of pre-liver transplantation TACE and post-transplant occurrence of hepatic artery complications.

The occurrence of hepatic artery complications after liver transplantation in patients treated with TACE could be related to mechanical effects of arterial instrumentation or to inflammatory changes of the arterial wall in response to infusion of chemotherapeutic or embolic agents. This hypothesis is supported by reports of structural injuries to the anastomotic site of the hepatic artery in a large proportion of patients after TACE. In 14\% of post-TACE cases alternative arterial reconstructions were needed to facilitate hepatic artery reperfusion. Despite frequent intra-operative salvage of arterial anastomosis during liver transplantation in TACE patients, our meta-analysis shows evidence of no more than a modest effect on post-procedural hepatic artery complications (point estimate: OR 1.6), clinically translating into an estimated increase of hepatic artery complications from $6 \%$ to $9 \%$ in TACE pre-treated patients, or affecting by estimation approximately 1:30 patients undergoing TACE prior to transplantation. The modest increased risk may reflect the fact that immediate 
recognition and adequate intra-operative salvage of the arterial anastomosis may successfully avert more severe postoperative sequelae.

Nevertheless, complications of the hepatic artery may have devastating consequences for the individual and lead to unnecessary waste and limited durability of scarce donor organs. A potential higher need for alternative arterial reconstructions may challenge the decision to accept high-risk grafts in TACE pre-treated recipients.

A previous study identified TACE as a risk factor for hepatic artery thrombosis, along with paediatric liver transplantation and aberrant arterial anatomy. ${ }^{29}$ Other risk factors for hepatic artery thrombosis reported in literature are CMV mismatch, re-transplantation and donor age. ${ }^{1,30}$ Recipient risk factors are infrequently identified as potential risk factors for hepatic artery complications, however, it is conceivable that age, history of smoking, diabetes, and atherosclerosis of the recipient may confer additional risks to the arterial revascularization. In our meta-analysis it was not possible to control for these risk factors.

While donor risk factors will not be known until transplantation, recipient risk factors and perceived quality of the artery at time of listing (such as a fragile artery on imaging or atherosclerosis at origin of the celiac trunk) may be taken into account and weighed against an additional compromise of arterial quality by choosing TACE as a bridging therapy.

No evidence of an association between pre-liver transplantation TACE and post transplant occurrence of biliary tract complications was found. However, data on biliary complications in included studies was frequently flawed by lack of a clear definition and variable inclusion of different post-transplant biliary problems. Hypothetically, risk of biliary tract complications after TACE would constitute indirect effects of a prolonged arterialisation phase during graft implantation or late effects secondary to hepatic artery complications. Given the multifactorial nature of biliary tract complications after transplantation, a potential 
indirect effect of TACE on biliary tract complications may be too small or clinically irrelevant. Moreover, adequate intra-operative interventions and post-operative recognition and management of hepatic artery complications may prevent development of subsequent biliary tract complications.

Our systematic review has several limitations. Although our analysis did not show heterogeneity, we assume that the studied populations are likely to exhibit some degree of variation, such as different tumour stages, use of different donor types, and variable waiting times with unknown numbers of waitlist dropouts. Additional heterogeneity may exist due to differences in TACE techniques between institutions, such as administered drugs, selectivity and number of TACE cycles and treatment schedules. Although the treatments algorithms used for TACE differ, the treatment concept including intra-arterial manipulation and administration of chemotherapeutic drugs remains the same. Furthermore, anastomotic techniques and postoperative anticoagulation protocols may differ between transplant centers. Due to patient selection and inconsistency in the reported data of individual studies, we were not able to take potential confounders into account, such as tumour factors, etiology of HCC, MELD scores, previous treatments and other risk factors for hepatic artery thrombosis. In addition, control groups of four studies contained patients with non-HCC related indications for liver transplantation and different severity of liver disease than the TACE treatment groups. ${ }^{10,16,18,20}$ Regarding the outcome parameters, the definitions of both hepatic artery and biliary tract complications used in the included studies were variable and not always clearly described. These limitations could cause over- or underestimation of our results and potentially reduce generalizability of our conclusions. Very limited data was available on timing of hepatic artery complications and for meta-analysis no distinction could be made between early vs. late hepatic artery complications. Finally, based on the available data we 
were not able to study the outcomes of hepatic artery complications on graft and patient survival and balance this against the benefits of TACE as a bridging strategy.

Waitlist dropout could not be assessed as an outcome parameter in the present review design. Because of the reluctance to withhold treatment in patients at risk of tumour progression beyond Milan criteria and subsequent heterogeneity of study populations, the actual effect of TACE on waitlist dropout is difficult to determine. Radiofrequency and microwave ablation (RFA/MWA) are alternative bridging strategies available for patients with smaller tumours depending on tumour location. ${ }^{34,35}$ The possibility of tumour resection and salvage liver transplantation could sometimes be considered, depending on patient and tumour characteristics. ${ }^{35}$ Neo-adjuvant Sorafenib has been explored in smaller studies as a bridge to transplantation, however, concerns are raised of increases in biliary complications and episodes of rejection following liver transplantation after Sorafenib use. ${ }^{43-45} \cdot{ }^{46,47}$. In summary, no equal alternative may be available for patients with larger HCC's.

Successfully down-staged patients seem to have similar survival after liver transplantation compared to patients that initially met Milan criteria and patients with complete pathologic tumour response have better survival after liver transplantation than those with incomplete responses. ${ }^{34,36-39}$ A selection bias of patients with favourable tumour biology may, however, play a role in positive results of TACE bridged patients. ${ }^{36,39}$ Oncologic and survival endpoints were not included in the present review. Only two studies in our meta-analyses presented long-term survival rates, and showed no association with TACE, hepatic artery or biliary tract complications. ${ }^{15,21}$ 
More data are needed to validate our conclusions and consolidate the hypothesis, however, given the large number of patients needed to detect a difference for outcomes with a low incidence rate, robust randomized controlled trials may be unlikely to succeed in the clinical setting of pre-liver transplantation TACE.

Future prospective studies focussing on microscopic damage to the arterial wall, intraoperative salvage of the arterial anastomosis and subsequent occurrence of post-operative arterial and biliary complications could provide insight in the potential causal pathway.

Whether the potential benefits of TACE as a bridging strategy to liver transplantation for liver transplant candidates with HCC outweigh TACE induced morbidity, could not be answered by the present review. Among the many trade-offs to balance the risks and benefits of such treatment, neo-adjuvant TACE might be considered an additional recipient risk factor for the development of hepatic artery complications after liver transplantation. A more challenging arterial reperfusion may be anticipated on acceptance of a graft in TACE recipients.

\section{$\underline{\text { Acknowledgements }}$}

We thank Wichor Bramer, biomedical information specialist of the medical library in the Erasmus Medical Center, for his expert assistance with the systematic literature search. The Peter Morris Centre for Evidence in Transplantation (PMCET) advised on sections of the paper as part of the collaboration between the European Society for Organ Transplantation (ESOT) and PMCET. 


\section{References}

1. Bekker J, Ploem S, De Jong K. Early hepatic artery thrombosis after liver transplantation: a systematic review of the incidence, outcome and risk factors. American Journal of Transplantation. 2009;9(4):746-757.

2. Heckman JT, Devera MB, Marsh JW, et al. Bridging locoregional therapy for hepatocellular carcinoma prior to liver transplantation. Ann Surg Oncol. 2008;15(11):3169-3177.

3. Marelli L, Stigliano R, Triantos C, Senzolo M. Transarterial therapy for hepatocellular carcinoma: which technique is more effective? A systematic review of cohort and randomized studies. Cardiovascular and .... 2007.

4. Golfieri R, Cappelli A, Cucchetti A, et al. Efficacy of selective transarterial chemoembolization in inducing tumor necrosis in small $(<5 \mathrm{~cm})$ hepatocellular carcinomas. Hepatology. 2011;53(5):1580-1589.

5. Loffroy R, Guiu B, Cercueil JP, Krause D. Endovascular therapeutic embolisation: an overview of occluding agents and their effects on embolised tissues. Curr Vasc Pharmacol. 2009;7(2):250-263.

6. Lewis AL, Holden RR. DC Bead embolic drug-eluting bead: Clinical application in the locoregional treatment of tumours. Expert Opin Drug Deliv. 2011;8(2):153-169.

7. Xia J, Ren Z, Ye S, et al. Study of severe and rare complications of transarterial chemoembolization (TACE) for liver cancer. Eur J Radiol. 2006;59(3):407-412.

8. Dhanasekaran R, Kooby DA, Staley CA, Kauh JS, Khanna V, Kim HS. Comparison of conventional transarterial chemoembolization (TACE) and chemoembolization with doxorubicin drug eluting beads (DEB) for unresectable hepatocelluar carcinoma (HCC). J Surg Oncol. 2010;101(6):476-480.

9. Malagari K, Pomoni M, Spyridopoulos TN, et al. Safety profile of sequential transcatheter chemoembolization with DC Bead: results of 237 hepatocellular carcinoma (HCC) patients. Cardiovasc Intervent Radiol. 2011;34(4):774-785.

10. Casadaban L, Malespin M, Cheung A, et al. Presurgical transarterial chemoembolization does not increase biliary stricture incidence in orthotopic liver transplant patients. Transplant Proc. 2014;46(5):1413-1419.

11. Goel A, Mehta N, Guy J, et al. Hepatic artery and biliary complications in liver transplant recipients undergoing pretransplant transarterial chemoembolization. Liver Transplant. 2014;20(10):1221-1228.

12. Kanakadandi V, Park J, Ahmad J. Chemoembolization therapy of hepatocellular carcinoma prior to liver transplant is associated with the development of posttransplant biliary anastomotic strictures. Hepatology. 2012;56:475A-476A.

13. $\mathrm{Li} \mathrm{H}, \mathrm{Li} \mathrm{B}$, Wei $\mathrm{Y}$, et al. Preoperative transarterial chemoembolization does not increase hepatic artery complications after liver transplantation: A single center 12year experience. Clin Res Hepatol Gastroenterol. 2015.

14. Lin TS, Chiang YC, Chen CL, et al. Intimal dissection of the hepatic artery following transarterial embolization for hepatocellular carcinoma: An intraoperative problem in adult living donor liver transplantation. Liver Transplant. 2009;15(11):1553-1556.

15. Majno PE, Adam R, Bismuth $\mathrm{H}$, et al. Influence of preoperative transarterial lipiodol chemoembolization on resection and transplantation for hepatocellular carcinoma in patients with cirrhosis. Ann Surg. 1997;226(6):688-703.

16. Mekeel KL, Moss AA, Reddy K, et al. The risk of hepatic arterial complications associated with trans-arterial chemoembolization prior to liver transplantation for hepatocellular carcinoma. Transplantation. 2010;90:781. 
17. Panaro F, Ramos J, Gallix B, et al. Hepatic artery complications following liver transplantation. Does preoperative chemoembolization impact the postoperative course? Clin Transplant. 2014;28(5):598-605.

18. Pravisani R, Baccarani U, Adani GL, et al. Transarterial chemoembolization does not harm the hepatic artery at transplantation. Transplant Int. 2015;28:212.

19. Ramanathan M, Pedersen M, Choi M, Seetharam AB. Downstaging or bridging therapy of hepatocellular cancer does not increase incidence of biliary complications after liver transplantation. Hepatology. 2015;62:833A.

20. Richard IHM, Silberzweig JE, Mitty HA, Lou WYW, Ahn J, Cooper JM. Hepatic arterial complications in liver transplant recipients treated with pretransplantation chemoembolization for hepatocellular carcinoma. Radiology. 2000;214(3):775-779.

21. Saborido BP, Meneu JC, Moreno E, Garcia I, Moreno A, Fundora Y. Is transarterial chemoembolization necessary before liver transplantation for hepatocellular carcinoma? Am J Surg. 2005;190(3):383-387.

22. Shiani A, Rodriguez A, Lipka S, et al. Incidence of biliary stricture after transarterial chemoembolization for hepatocellular carcinoma in orthotopic liver transplant patients. Am J Gastroenterol. 2015;110:S907-S908.

23. Shiani A, Rodriguez A, Lipka S, et al. Vascular complications after transarterial chemoembolization in orthotopic liver transplant patients. Am J Gastroenterol. 2015;110:S906.

24. Deeks J, Higgins J, Altman D, Green S. Cochrane handbook for systematic reviews of interventions version 5.1. 0 (updated March 2011). The Cochrane Collaboration. 2011.

25. Moher D, Shamseer L, Clarke M, et al. Preferred reporting items for systematic review and meta-analysis protocols (PRISMA-P) 2015 statement. Systematic reviews. 2015;4(1):1.

26. Downs SH, Black N. The feasibility of creating a checklist for the assessment of the methodological quality both of randomised and non-randomised studies of health care interventions. J Epidemiol Community Health. 1998;52(6):377-384.

27. Wallace BC, Dahabreh IJ, Trikalinos TA, Lau J, Trow P, Schmid CH. Closing the gap between methodologists and end-users: R as a computational back-end. J Stat Softw. 2012;49(5):1-15.

28. Lopez GD, Blanco FG, De Alarcon LF, et al. Early hepatic artery complications following liver transplantation in patients undergoing preoperative doxorubicineluting bead transarterial chemoembilization. Transplant Int. 2015;28:474.

29. Duffy JP, Hong JC, Farmer DG, et al. Vascular complications of orthotopic liver transplantation: experience in more than 4,200 patients. $J$ Am Coll Surg. 2009;208(5):896-903; discussion 903-895.

30. Silva MA, Jambulingam PS, Gunson BK, et al. Hepatic artery thrombosis following orthotopic liver transplantation: a 10-year experience from a single centre in the United Kingdom. Liver Transpl. 2006;12(1):146-151.

31. Graziadei IW, Sandmueller H, Waldenberger P, et al. Chemoembolization followed by liver transplantation for hepatocellular carcinoma impedes tumor progression while on the waiting list and leads to excellent outcome. Liver Transpl. 2003;9(6):557-563.

32. Lesurtel M, Mullhaupt B, Pestalozzi BC, Pfammatter T, Clavien PA. Transarterial chemoembolization as a bridge to liver transplantation for hepatocellular carcinoma: An evidence-based analysis. Am J Transplant. 2006;6(11):2644-2650.

33. Clavien PA, Lesurtel M, Bossuyt PM, Gores GJ, Langer B, Perrier A. Recommendations for liver transplantation for hepatocellular carcinoma: an international consensus conference report. Lancet Oncol. 2012;13(1):e11-22. 
34. Fujiki M, Aucejo F, Choi M, Kim R. Neo-adjuvant therapy for hepatocellular carcinoma before liver transplantation: where do we stand? World J Gastroenterol. 2014;20(18):5308-5319.

35. Majno P, Lencioni R, Mornex F, Girard N, Poon RT, Cherqui D. Is the treatment of hepatocellular carcinoma on the waiting list necessary? Liver Transpl. 2011;17 Suppl 2:S98-108.

36. Yao FY, Fidelman N. Reassessing the boundaries of liver transplantation for hepatocellular carcinoma: Where do we stand with tumor down - staging? Hepatology. 2016;63(3):1014-1025.

37. Allard MA, Sebagh M, Ruiz A, et al. Does pathological response after transarterial chemoembolization for hepatocellular carcinoma in cirrhotic patients with cirrhosis predict outcome after liver resection or transplantation? J Hepatol. 2015;63(1):83-92.

38. Ravaioli M, Grazi GL, Piscaglia F, et al. Liver transplantation for hepatocellular carcinoma: results of down-staging in patients initially outside the Milan selection criteria. Am J Transplant. 2008;8(12):2547-2557.

39. EASL-EORTC clinical practice guidelines: management of hepatocellular carcinoma. Eur J Cancer. 2012;48(5):599-641.

40. Llovet JM, Mas X, Aponte JJ, et al. Cost effectiveness of adjuvant therapy for hepatocellular carcinoma during the waiting list for liver transplantation. Gut. 2002;50(1):123-128.

41. Andreou A, Gul S, Pascher A, et al. Patient and tumour biology predict survival beyond the Milan criteria in liver transplantation for hepatocellular carcinoma. $H P B$ (Oxford). 2015;17(2):168-175.

42. Agopian VG, Harlander-Locke M, Zarrinpar A, et al. A novel prognostic nomogram accurately predicts hepatocellular carcinoma recurrence after liver transplantation: analysis of 865 consecutive liver transplant recipients. $J$ Am Coll Surg. 2015;220(4):416-427.

43. Vitale A, Volk ML, Pastorelli D, et al. Use of sorafenib in patients with hepatocellular carcinoma before liver transplantation: a cost-benefit analysis while awaiting data on sorafenib safety. Hepatology. 2010;51(1):165-173.

44. Hoffmann K, Ganten T, Gotthardtp D, et al. Impact of neo-adjuvant Sorafenib treatment on liver transplantation in HCC patients - a prospective, randomized, double-blind, phase III trial. BMC Cancer. 2015;15:392.

45. Kulik L, Vouche M, Koppe S, et al. Prospective randomized pilot study of Y90+/sorafenib as bridge to transplantation in hepatocellular carcinoma. J Hepatol. 2014;61(2):309-317.

46. Truesdale AE, Caldwell SH, Shah NL, et al. Sorafenib therapy for hepatocellular carcinoma prior to liver transplant is associated with increased complications after transplant. Transpl Int. 2011;24(10):991-998.

47. Frenette CT, Boktour M, Burroughs SG, et al. Pre-transplant utilization of sorafenib is not associated with increased complications after liver transplantation. Transpl Int. 2013;26(7):734-739. 
Tables

Table 1

Quality assessment according to the Downs and Black checklist.

\begin{tabular}{|c|c|c|c|c|c|c|c|c|}
\hline . & & 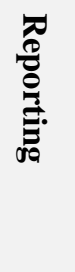 & 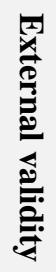 & 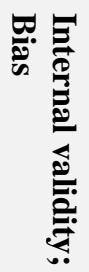 & 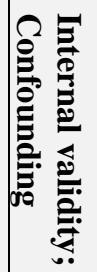 & $\stackrel{0}{0}$ & $\begin{array}{l}\overrightarrow{0} \\
\vec{\theta} \\
\vec{E} \\
\vec{E}\end{array}$ & 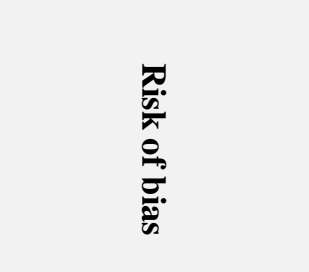 \\
\hline \multirow{8}{*}{ 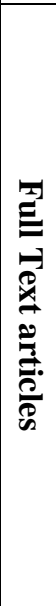 } & Casadaban et al. ${ }^{10}$ & 10 & 1 & 5 & 2 & 0 & 18 & $\begin{array}{c}\text { Risk for selection } \\
\text { bias }\end{array}$ \\
\hline & Goel et al. ${ }^{11}$ & 8 & 3 & 4 & 3 & 5 & 23 & Low risk \\
\hline & Li et al. ${ }^{13}$ & 6 & 1 & 4 & 2 & 0 & 13 & $\begin{array}{l}\text { Risk for selection } \\
\text { bias }\end{array}$ \\
\hline & Lin et al. ${ }^{14}$ & 4 & 3 & 4 & 2 & 0 & 13 & $\begin{array}{c}\text { Risk for reporting } \\
\text { bias }\end{array}$ \\
\hline & Majno et al. ${ }^{15}$ & 9 & 3 & 3 & 2 & 0 & 17 & $\begin{array}{c}\text { Risk for reporting } \\
\text { bias }\end{array}$ \\
\hline & Panaro et al. ${ }^{17}$ & 9 & 3 & 6 & 2 & 0 & 20 & $\begin{array}{c}\text { Risk for reporting } \\
\text { bias }\end{array}$ \\
\hline & Richard et al. ${ }^{20}$ & 6 & 1 & 4 & 2 & 0 & 13 & $\begin{array}{l}\text { Risk for selection } \\
\text { bias }\end{array}$ \\
\hline & Saborido et al. ${ }^{21}$ & 9 & 3 & 2 & 2 & 0 & 16 & Risk of reporting bias \\
\hline \multirow{6}{*}{ 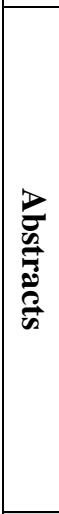 } & $\begin{array}{l}\text { Kanakadandi et al. } \\
12\end{array}$ & 5 & 3 & 3 & 2 & 0 & 13 & $\begin{array}{l}\text { Risk for selection } \\
\text { bias } \\
\end{array}$ \\
\hline & Mekeel et al. ${ }^{16}$ & 5 & 3 & 3 & 2 & 0 & 13 & $\begin{array}{c}\text { Risk for selection } \\
\text { bias }\end{array}$ \\
\hline & Pravisani et al. ${ }^{18}$ & 8 & 1 & 4 & 1 & 0 & 14 & $\begin{array}{c}\text { Risk for } \\
\text { selection/reporting } \\
\text { bias }\end{array}$ \\
\hline & $\begin{array}{l}\text { Ramanathan et al. } \\
19\end{array}$ & 3 & 1 & 3 & 1 & 0 & 8 & Low risk \\
\hline & Shiani et al. ${ }^{22}$ & 6 & 1 & 2 & 1 & 0 & 10 & $\begin{array}{c}\text { Risk for reporting } \\
\text { bias }\end{array}$ \\
\hline & Shiani et al. ${ }^{23}$ & 6 & 1 & 2 & 1 & 0 & 10 & Low risk \\
\hline \multicolumn{2}{|r|}{ Maximum score } & 11 & 3 & 7 & 6 & 5 & 32 & \\
\hline
\end{tabular}




\section{Table 2}

Characteristics of the included studies

Legend: DOX, doxorubicin; MMC, mitomycin C; CIS, cisplatin; NR, not reported; AS, anastomostic biliary strictures; NAS, non-anastomotic biliary strictures.

\begin{tabular}{|c|c|c|c|c|c|c|c|c|c|c|c|c|}
\hline & $\stackrel{0}{E}$ & $\underset{\overbrace{}}{\mathscr{3}}$ & 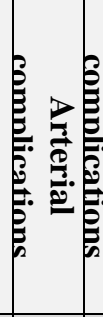 & : & $\stackrel{3}{3}$ & 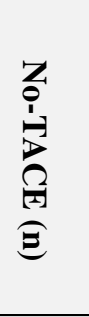 & 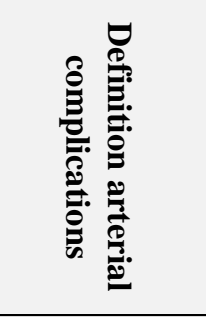 & 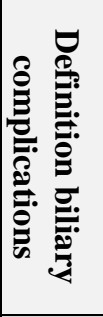 & 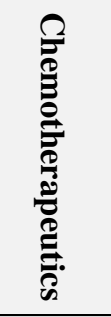 & 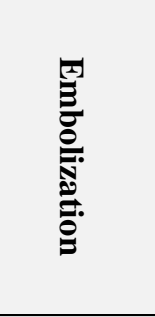 & 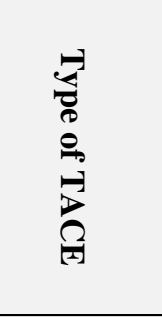 & 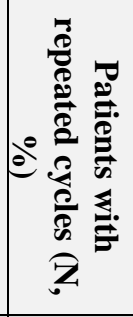 \\
\hline \multirow{8}{*}{ 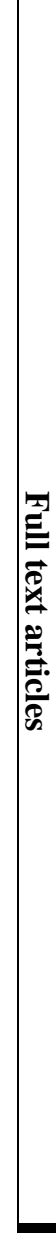 } & Casadaban ${ }^{10}$ & 2014 & & $\checkmark$ & 46 & 186 & & $\begin{array}{l}\text { AS, } \\
\text { NAS }\end{array}$ & $\begin{array}{l}\text { DOX } \\
\text { MMC } \\
\text { CIS }\end{array}$ & $\begin{array}{l}\text { Drug } \\
\text { eluting } \\
\text { beads }\end{array}$ & $\begin{array}{l}\text { Segmental/ } \\
\text { Selective }\end{array}$ & NR \\
\hline & Goel ${ }^{11}$ & 2014 & $\checkmark$ & $\checkmark$ & 328 & 128 & $\begin{array}{c}\text { Thrombosis, } \\
\text { Stenosis, } \\
\text { Pseudo- } \\
\text { aneurysm }\end{array}$ & $\begin{array}{l}\text { NAS } \\
\text { Bile } \\
\text { leaks }\end{array}$ & $\begin{array}{l}\text { DOX } \\
\text { MMC } \\
\text { CIS }\end{array}$ & $\begin{array}{l}\text { Ethiodized } \\
\text { oil }\end{array}$ & Selective & $\begin{array}{l}105 \\
32 \%\end{array}$ \\
\hline & $\mathbf{L i}^{13}$ & 2015 & $\checkmark$ & & 111 & 339 & $\begin{array}{c}\text { Thrombosis, } \\
\text { stenosis, } \\
\text { pseudo- } \\
\text { aneurysm } \\
\end{array}$ & & $\begin{array}{l}\text { DOX } \\
\text { Metuxi } \\
\text { mab }\end{array}$ & $\begin{array}{l}\text { polyvinyl } \\
\text { alcohol }\end{array}$ & Selective & $75,68 \%$ \\
\hline & $\operatorname{Lin}^{14}$ & 2009 & $\checkmark$ & & 40 & 14 & NR & & NR & NR & NR & $17,43 \%$ \\
\hline & Majno $^{15}$ & 1997 & $\checkmark$ & $\checkmark$ & 54 & 57 & NR & NR & DOX & $\begin{array}{l}\text { Gelatin } \\
\text { sponge }\end{array}$ & Segmental & $15,28 \%$ \\
\hline & Panaro ${ }^{17}$ & 2014 & $\checkmark$ & $\checkmark$ & 32 & 35 & NR & NR & DOX & $\begin{array}{c}\text { Drug } \\
\text { eluting } \\
\text { beads }\end{array}$ & Selective & NR \\
\hline & Richard 20 & 2000 & $\checkmark$ & & 47 & 1154 & $\begin{array}{l}\text { Thrombosis, } \\
\text { stenosis, } \\
\text { pseudo- } \\
\text { aneurysm, } \\
\text { anastomotic } \\
\text { disruption } \\
\end{array}$ & & $\begin{array}{l}\text { DOX } \\
\text { MMC } \\
\text { CIS }\end{array}$ & $\begin{array}{l}\text { Collagen, } \\
\text { gelatine } \\
\text { sponge, } \\
\text { polyvinyl } \\
\text { alcohol }\end{array}$ & Selective & $16,34 \%$ \\
\hline & Saborido ${ }^{21}$ & 2005 & $\checkmark$ & & 18 & 28 & $\begin{array}{c}\text { Thrombosis or } \\
\text { other } \\
\text { complications }\end{array}$ & & DOX & Gelfoam & NR & 0 \\
\hline \multirow{6}{*}{ 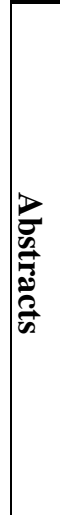 } & $\begin{array}{l}\text { Kanakadandi } \\
12\end{array}$ & 2012 & & $\checkmark$ & 38 & 14 & & AS & NR & NR & NR & $24,63 \%$ \\
\hline & Mekeel ${ }^{16}$ & 2010 & $\checkmark$ & & 76 & 310 & $\begin{array}{c}\text { Thrombosis, } \\
\text { stenosis }\end{array}$ & & NR & NR & NR & NR \\
\hline & Pravisani ${ }^{18}$ & 2015 & $\checkmark$ & & 73 & 198 & NR & & NR & NR & NR & NR \\
\hline & $\begin{array}{l}\text { Ramanathan } \\
19\end{array}$ & 2015 & & $\checkmark$ & 82 & 198 & & $\begin{array}{l}\text { NA, } \\
\text { bile } \\
\text { leak }\end{array}$ & NR & NR & NR & NR \\
\hline & Shiani ${ }^{22}$ & 2015 & & $\checkmark$ & 58 & 31 & & NR & NR & NR & NR & NR \\
\hline & Shiani ${ }^{23}$ & 2015 & $\checkmark$ & & 58 & 31 & Stenosis & & NR & NR & NR & NR \\
\hline
\end{tabular}




\section{Table 3}

Hepatic artery complications

\begin{tabular}{|c|c|c|c|c|}
\hline & \multicolumn{2}{|r|}{ TACE } & \multicolumn{2}{|r|}{ No-TACE } \\
\hline Study & $\mathbf{N}$ & Complications (N, \%) & $\mathbf{N}$ & Complications $(\mathrm{N}, \%)$ \\
\hline Goel ${ }^{11}$ & 328 & $\begin{array}{r}\text { Thrombosis: } 12(3.7 \%) \\
\text { Stenosis: } 14(4.3 \%) \\
\text { Pseudoaneurysm: } 1(0.3 \%)\end{array}$ & 128 & $\begin{array}{r}\text { Thrombosis: } 5(3.9 \%) \\
\text { Stenosis: } 0(0.0 \%) \\
\text { Pseudoaneurysm: } 1(0.8 \%)\end{array}$ \\
\hline $\mathbf{L i}^{13}$ & 111 & $\begin{array}{r}\text { Thrombosis: } 4(3.6 \%) \\
\text { Aneurysm: } 1(0.9 \%)\end{array}$ & 339 & $\begin{array}{r}\text { Thrombosis: } 2(0.6 \%) \\
\text { Stenosis: } 3(0.9 \%)\end{array}$ \\
\hline $\operatorname{Lin}^{14}$ & 40 & Thrombosis: $2(5.0 \%)$ & 14 & None \\
\hline Majno ${ }^{15}$ & 54 & NS arterial complication: $2(3.7 \%)$ & 57 & NS arterial complication: $2(3.5 \%)$ \\
\hline Mekeel ${ }^{16}$ & 76 & $\begin{array}{l}\text { Thrombosis: } 1(1.3 \%) \\
\text { Stenosis: } 8(10.5 \%)\end{array}$ & 310 & $\begin{array}{r}\text { Thrombosis: } 12(3.9 \%) \\
\text { Stenosis: } 21(6.8 \%)\end{array}$ \\
\hline Panaro ${ }^{17}$ & 32 & $\begin{array}{r}\text { Thrombosis: } 1(3.1 \%) \\
\text { Stenosis: } 3(9.4 \%) \\
\text { Pseudoaneurysm: } 1(3.1 \%) \\
\text { Arterial dissection: } 2(6.3 \%)\end{array}$ & 35 & $\begin{array}{r}\text { Thrombosis: } 1(2.9 \%) \\
\text { Stenosis: } 1(2.9 \%) \\
\text { Stenosis \& Pseudoaneurysm:1 }(2.9 \%)\end{array}$ \\
\hline Pravisani ${ }^{18}$ & 73 & $\begin{array}{r}\text { Thrombosis: } 2(2.7 \%) \\
\text { Stenosis: } 5(6.8 \%) \\
\text { Pseudoaneurysm: } 1(1.4 \%) \\
\end{array}$ & 198 & $\begin{array}{r}\text { Thrombosis: } 6(3.0 \%) \\
\text { Stenosis: } 14(7.1 \%) \\
\text { Pseudoaneurysm: } 7(3.5 \%)\end{array}$ \\
\hline Richard $^{20}$ & 47 & $\begin{array}{r}\text { Thrombosis: } 4(8.5 \%) \\
\text { Pseudoaneurysm: } 2(4.3 \%)\end{array}$ & 1154 & $\begin{array}{r}\text { Thrombosis: } 62(5.4 \%) \\
\text { NS arterial complication: } 6(0.5 \%)\end{array}$ \\
\hline Saborido $^{21}$ & 18 & None & 28 & None \\
\hline Shiani ${ }^{23}$ & 58 & Stenosis: $12(20.7 \%)$ & 31 & Stenosis: $3(9.7 \%)$ \\
\hline
\end{tabular}

Legend: NS, not specified 


\section{Table 4}

Biliary tract complications

\begin{tabular}{|c|c|c|c|c|}
\hline & \multicolumn{2}{|r|}{ TACE } & \multicolumn{2}{|r|}{ No TACE } \\
\hline Study & $\mathbf{N}$ & Complications (N, \%) & $\mathbf{N}$ & Complications (N, \%) \\
\hline Casaban ${ }^{10}$ & 46 & AS+NAS: $14(30.4 \%)$ & 186 & AS+NAS: $57(30.6 \%)$ \\
\hline Goel $^{11}$ & 328 & $\begin{array}{r}\text { NAS: } 51(15.6 \%) \\
\text { Bile leak: } 10(3.0 \%) \\
\text { Diffuse biliary injury: } 4(1.2 \%)\end{array}$ & 128 & $\begin{array}{c}\text { NAS: } 17(13.3 \%) \\
\text { Bile leak: } 5(3.9 \%)\end{array}$ \\
\hline Kanakadandi ${ }^{12}$ & 38 & AS: $21(55.3 \%)$ & 14 & AS: $5(35.7 \%)$ \\
\hline Majno ${ }^{15}$ & 54 & NS Biliary complication: $8(14.8 \%)$ & 57 & NS biliary complication: $10(17.5 \%)$ \\
\hline Panaro ${ }^{17}$ & 32 & None & 35 & None \\
\hline Ramanathan 19 & 82 & NAS/bile leak: 32 (39\%) & 198 & NAS/bile leak: 43 (21.7\%) \\
\hline Shiani ${ }^{22}$ & 58 & $\begin{array}{r}\text { AS: } 9(15.5 \%) \\
\text { NAS: } 3(5.2 \%)\end{array}$ & 31 & $\begin{array}{r}\text { AS: } 5(16.1 \%) \\
\text { NAS: } 1(3.2 \%)\end{array}$ \\
\hline
\end{tabular}

Legend: NS, not specified; AS, anastomostic biliary strictures; NAS, non-anastomotic biliary strictures 
Title and legends for figures

\section{Figure 1}

PRISMA flowchart

\section{Figure 2}

Forest plot hepatic artery complications

\section{Figure 3}

Forest plot hepatic artery thrombosis

\section{Figure 4}

Forest plot biliary tract complications 